\title{
Time-resolved hyperfast processes of strongly correlated electrons during the coherent excitation and decay of multiply excited and inner-hole excited states
}

\author{
Th. Mercouris, ${ }^{1, *}$ Y. Komninos, ${ }^{1, \dagger}$ and C. A. Nicolaides ${ }^{1,2, \$}$ \\ ${ }^{1}$ Theoretical and Physical Chemistry Institute, National Hellenic Research Foundation, 48 Vasileos Constantinou Avenue, \\ Athens, 11635 Greece \\ ${ }^{2}$ Physics Department, National Technical University, Athens, 15780 Greece
}

(Received 9 May 2007; published 27 September 2007)

\begin{abstract}
In the framework of the state-specific expansion approach, we have solved nonperturbatively the timedependent Schrödinger equation for systems involving the time-dependent excitation of nonstationary states by hypershort one or two electromagnetic pulses. The related problems concern the quantitative determination of the consequences of strong electron correlation in excited atomic states of the discrete or of the continuous spectrum. As prototypical applications we chose the time-resolved coherent excitation and decay of the $2 s$-hole $1 s^{2} 2 s 2 p^{6} 3 s^{2} 3 p^{1,3} P^{0}$ Auger states of aluminum, where dominant channels representing one- as well as twoelectron continua are taken into account, and the preparation of nonstationary states and the subsequent electron correlation beats in $\mathrm{Al}$ and in $\mathrm{N}^{3+}$. Calculations for the problem of coherent inner-hole ionization and Auger decay in $\mathrm{Al}$ account for the effect of interference coming from the presence of the channel of direct two-electron ionization. The result is that for the $\mathrm{Al}^{+}{ }^{1} P^{0}$ channel the Auger electron distribution is asymmetric. On the contrary, for the $\mathrm{Al}^{+3} \mathrm{P}^{0}$ channel the contribution of the direct two-electron ionization is weak and the corresponding Auger distribution is essentially symmetric. The intensities used were weak, so as to avoid shifts and to render high order processes beyond those in resonance or near-resonance essentially negligible. As a result, the transition probabilities are very small, a result that ought to guide experimental work. For example, for intensity $8.75 \times 10^{11} \mathrm{~W} / \mathrm{cm}^{2}$ the percentage of initial state population that ionizes is $0.02 \%$. In spite of the overall reasonable approximations and size reductions, for the Al problem the system of coupled integrodifferential equations that had to be solved contains more than 300,000 basis wave functions.
\end{abstract}

DOI: 10.1103/PhysRevA.76.033417

PACS number(s): 32.80.Rm, 31.15.Ar, 31.25.Jf, 42.65.Re

\section{INTRODUCTION}

Recent results of ab initio calculations that solve nonperturbatively the time-dependent Schrödinger equation (TDSE) describing the interaction of real atomic states with electromagnetic pulses of short wavelength (of the order of tens of $\mathrm{eV}$ or higher), and of short and hypershort duration [of the order of tens of femtoseconds (fs) down to attoseconds (as)], indicate that it is possible to prepare excited nonstationary atomic states in such a way as to be able to identify quantitatively effects of electron correlation and rearrangement (e.g., autoionization) that are time-resolved within hyperfast, but experimentally feasible, time ranges [1-4]. The theory and calculations of [1-4] were concerned with problems that were formulated around the spectrum of the helium atom, with the initial state being either the ground state or one of the low-lying excited discrete ones.

As regards our work [1-3], the first two papers, [1,2], dealt mainly with the exploration of the possibility of coherently exciting and of time-resolving on the attosecond scale effects of superpositions of states that are coupled via strong electron correlations. Quantitative results on time-resolved quantities such as electron correlation beats (ECB), autoionization, or geometrical rearrangements of electron pairs were reported. Paper [3] analyzed systematically and quantita-

\footnotetext{
*thmerc@eie.gr

†ykomn@eie.gr

*aan@eie.gr
}

tively the time resolution of the formation of the asymmetric photoabsorption profile of the $\mathrm{He} 2 s 2 p^{1} P^{0}$ resonance state, a possible phenomenon whose relevance to attosecond physics was proposed by Wickenhauser et al. [5]. The basic theoretical framework and computational tool for these types of computations and analyses were introduced in [6], under the name of "state-specific expansion approach" (SSEA).

Over the years, quantitative results of ab initio calculations regarding the nonperturbative time-dependent description of the interaction of laser pulses with atoms have been mostly restricted to the use of models or of one-electron approximations. However, as in the case of the less complex time-independent many-electron problem for stationary properties, it is preferable that the subject of the $a b$ initio solution of the TDSE for pulse-induced processes is tackled at a more sophisticated and reliable level. Furthermore, it is obviously desirable for the theoretical method to be applicable in a practical and economic way to any type of initial state and not just to states whose zero order wave functions are single determinantal, as are the cases of, say, $\mathrm{He}$ or $\mathrm{Mg}$. (References for such methods can be found in $[4,6])$. This is clearly a desideratum for experiment as well, if progress is to be characterized by new information on a variety of real systems. For example, only a small part of what one learns about control or about the effects of electron correlation from the experimental and/or theoretical studies of the interaction of the $\mathrm{He} 1 s^{2}{ }^{1} S$ ground state with a strong electromagnetic pulse, can be transferred to other systems. In other words, just as in the case of the decades-old time-independent many-electron problem and the chasm between the many- 
body problem for $\mathrm{He}$ and that for other larger atoms, especially in excited states, even a good calculation on the system "laser pulse- $\mathrm{He} 1 s^{2}{ }^{1} S$ " cannot help very much in understanding quantitatively field-induced phenomena exhibited by arbitrary ground or excited states of other atoms across the Periodic Table.

The present paper constitutes yet another type of contribution to the nascent field of theoretical-computational hyperfast time-resolved spectroscopy of highly excited (wavepacket) states in the discrete and the continuous spectrum in which the multifaceted effects of electronic structures and of electron correlation can be probed. This time, the atomic systems have more than two electrons. In the context of the SSEA, one of the added complexities compared to those of the He calculations is due to the requirement of handling the solution of time-dependent many-electron problems (TDMEPs) that involve the simultaneous presence of single- and double-electron continua. On the other hand, the issue of accounting for the term-dependent electronic structures and localized electron correlations in polyelectronic systems does not constitute an intrinsic and practical difficulty, since this problem has already been solved in the context of the SSEA [6].

As in previous applications of the SSEA, no empirically fit models are used in order to achieve the quantitative solutions of the relevant TDMEPs. We expect that, in view of the validity of the herein overall methodology for understanding and solving many-electron problems to a physically reliable degree of accuracy, the new results ought to provide useful information as to the scales of time resolution of the various effects of strongly correlating electrons in the discrete and the continuous spectra.

The problems that were tackled are described in Sec. III and the corresponding results are included in Sec. IV. We start in the next section by briefly reviewing and justifying elements of the SSEA.

\section{BRIEF REVIEW OF KEY FEATURES OF THE STATE-SPECIFIC EXPANSION APPROACH TO THE SOLUTION OF THE TIME-DEPENDENT SCHRÖDINGER EQUATION}

The SSEA implements basic quantum mechanical formalism and draws from knowledge on the solution of the timeindependent Schrödinger equation (TISE) for stationary states of the discrete and of the continuous spectrum.

Specifically, the proposal in the SSEA is to capitalize on the experience of computing state-specific nonrelativistic or relativistic wave functions for stationary states. These include wave functions with simple as well as with complex electronic structures, such as the heavily mixed valenceRydberg-scattering states (where autoionizing states also belong). According to the theory and justification published elsewhere, e.g., [7-10] and references therein, the form of these wave functions is compact and essentially the same, irrespective of the states that they represent. This fact renders their use in solving the TDSE (without unnecessary discounts), practical and economic. Thus, the nonperturbative solution of the TDSE is obtained (via a robust Taylor expan- sion technique [6]), by converting the linear TDSE into a system of coupled integrodifferential equations that result from the substitution in the TDSE of the expansion

$$
\Psi_{S S E A}(t)=\sum_{m} a_{m}(t) \Psi_{m}(q)+\int_{0} d E c_{E}(t) U_{E}\left(q^{\prime}, r\right) .
$$

The $\Psi_{m}(q)$ are state-specific square-integrable wave functions representing the discrete states and the localized components of the inner-hole or multiply excited resonance states, and the $U_{E}$ are scattering wave functions normalized to $\delta\left(E-E^{\prime}\right)$. The symbols $q, q^{\prime}$ stand collectively for the coordinates of the bound electrons. The symbol $r$ stands for the coordinate(s) of the free electron(s).

The wave functions in Eq. (1) are expressed as superposition of configurations (SoC), where both numerical and analytic orbitals, suitably optimized, enter. It is pointed out that the form (1) and its utilization in the SSEA methodology is also valid and practical in the relativistic electronic structure framework [11] that may be necessary for electron dynamics taking place in atomic fields with high effective nuclear charges.

It is now important to explain and justify the choice and computation of the terms in Eq. (1), since this is fundamental to the overall solution of the various TDMEPs.

The square integrable wave functions representing the discrete states and the localized parts of the autoionizing states over which the system evolves are obtained as a superposition of symmetry-adapted configurations in the form

$$
\Psi(q)=c_{0} \Phi_{0}(q)+\sum_{n} c_{n} \Phi_{n}(q) .
$$

The $\Phi_{0}(q)$ is an analytic or numerical state-specific HartreeFock (HF) or multiconfigurational HF (MCHF) squareintegrable wave function, constituting the zero order approximation to the exact eigenfunction. The choice depends on the state under consideration. The terms in $\Sigma_{n} c_{n} \Phi_{n}(q)$ represent the remaining contributions from interelectronic interactions that contribute to the energy and the squareintegrability of $\Psi(q)$. Their choice is based on theory and their calculation is carried out variationally in terms of analytic correlation orbitals, normally via nonorthonormal configuration interaction $(\mathrm{CI})$.

The form (2) is known from the very large number of publications on the time-independent many-electron problem for ground or low-lying excited stationary states of atoms and molecules. The difficulties in this area are mainly those of computational logistics regarding the possibility of processing huge numbers of determinantal basis functions representing electron virtual excitations and of related interaction integrals.

On the other hand, for the infinity of unperturbed Rydberg and multiply excited states, and, especially for the perturbed such states and for various types of resonances in the continuous spectrum, the use of the form (2) had to be justified and tested within frameworks of theories and methods that differ significantly from those that are suitable for, say, the ground states. For such excited states, the form (2) may present formal and practical difficulties that have to be tack- 
led reliably and consistently rather than be swept under the rug. For example, problems and questions arise that have to do with the presence of a finite or an infinite number of states of the same symmetry below the ones of interest, or with the presence of near degeneracies with other bound configurations and with one or more scattering continua. Anyway, the use of numerically computed HF or MCHF wave functions for excited discrete states and for inner-hole (multiply excited) autoionizing states may today be regarded as a reasonably understood approach to the zero order description of resonance states. To this purpose, one may use, as we do, the code published by Froese-Fischer [12]. Yet, this choice does not guarantee easy or successful convergence, considering, among other things, the fact that the HF equations are derived from variational theory and that most of the quasilocalized excited states in the continuous spectrum, whether observed yet or not, must be labeled by a superposition of strongly mixing open subshell configurations. In fact, about three to four decades ago, the use of HF-based polyelectronic theories and calculations were confined to ground or, in a few exceptions, to low-lying excited discrete states.

It is then evident that the adoption of the form (2) for such states must be justified as regards the feasibility of the calculation of both $\Phi_{0}(q)$ and $\Sigma_{n} c_{n} \Phi_{n}(q)$ of Eq. (2) in all cases. For resonance (autoionizing) states, the incorporation of the many-electron form (2) into a theory and computational treatment was introduced in [7] (see also [8-10] for extensions and various applications), by focusing on the existence of $N$-electron state-specific localized solutions (notwithstanding the possibility of different solutions due to multiple local energy minima).

The end result of the research program of combining state-specific many-electron methodology with theory that is appropriate for highly excited states in the discrete and in the continuous spectrum, is the practical possibility of analysis and efficient computation of a variety of properties and processes involving $N$-electron closed or open (sub)shell states, where $N$ may be larger than two. As an example, we mention the treatment of highly mixed excited states that was initiated in the early 1980s, whereby the state-specific form (2) was incorporated into theories of diffuse resonance states with open subshells and large widths, e.g., [13], and of perturbed spectra via a CI-based multichannel quantum defect theory $[10,14]$. As in Seaton's theory of the quantum defect [15], which depends on a pair of analytic forms of regular and irregular Coulomb functions, the theory of $[10,14]$ produces energies, quantum defects, and phase shifts. In addition, it produces $\mathrm{SoC}$ wave functions that are directly usable in CItype calculations of matrix elements of the Hamiltonian and of other operators.

Having obtained the square integrable $\Phi_{0}(q)$, the $\Sigma_{n} c_{n} \Phi_{n}(q)$ term is computable variationally. Depending on the symmetry, position, and structure of the state, the $\Phi_{n}(q)$ may or may not require serious computation of electron correlation. For example, if a numerical procedure is adopted, for unperturbed Rydberg states of high quantum numbers the important difficulty comes only from the necessity of numerical accuracy in the asymptotic region, unless the core is "soft" and influenced by electron correlations. If $\Phi_{0}(q)$ is a numerical MCHF wave function [12], as is normally the case with our state-specific computations, the correlation configurations $\Phi_{n}(q)$ contain numerical MCHF orbitals as well as energy-optimized analytic virtual orbitals. Here we note that for states without problems of strong near degeneracies, the form (2) can in principle be replaced by a large expansion of a purely MCHF wave function. For example, such large expansions were employed by Brage, Froese-Fischer, and Vaeck for the computation of energies and golden-rule widths of a few two- and three-electron autoionizing states [16].

Finally, there is the category of the $U_{E}\left(q^{\prime}, r\right)$ scattering wave functions, which constitute the orthonormal basis for the continuous spectrum. In our work, these are energynormalized numerical functions. Their core is, in general, represented by a sufficiently correlated bound wave function, to which the scattering orbital of the free electron(s) is coupled to form a symmetry-adapted $N$-electron scattering wave function. The scattering orbital is obtained numerically at each desired value of the free electron energy, by solving the relevant frozen core HF equations, as prescribed by Bates [17]. The core may be a single configuration or may contain one or two major correlation corrections. When interchannel coupling has to be accounted for, CI in the framework of $K$-matrix scattering formalism can be applied, using as basis sets the $U_{E}\left(q^{\prime}, r\right)[10,14]$.

As regards the atom-field interaction, normally the electric dipole approximation is used. (However, see [18] for theory and applications involving the full multipolar interaction Hamiltonian.)

As the time integration of the TDSE proceeds, i.e., as the determination as a function of time of all the coefficients in Eq. (1) proceeds, the resulting $\Psi_{S S E A}(t)$ is obtained at the level of accuracy that is appropriate for each problem of interest. A good calculation yields a $\Psi_{S S E A}(t)$ that contains the physically important information for the self-consistent fields of each stationary state and for the corresponding near degeneracies, electron correlations, and multichannel couplings. Convergence is tested by varying the total number of the states in Eq. (1), especially with respect to the range and density of the energy-discretized continuum.

Once $\Psi_{S S E A}(t)$ is obtained, its form is such that

(a) It is directly usable for further computation and analysis. For example, it can easily be projected on appropriately chosen stationary state wave functions in order to determine observable quantities.

(b) It exhibits clearly the interplay between dynamics and the electronic structures of the states over which the system evolves. The time-dependent coefficient of each statespecific wave function, whether in the discrete or in the continuous spectrum, can be reliably interpreted as a probability amplitude for that state, according to the rules of quantum mechanics.

\section{STATES AND EXCITATION-DECAY SCHEMES STUDIED IN THIS WORK}

The laser pulse-induced coherent excitation-decay schemes on which we report here are described in (i) and (ii) below. For case (i), the excitation step is induced by the 
simultaneous application of two pulses. Trial calculations indicated that, for the parameters of these problems, phase variation does not have a significant effect on the results.

(i) Two prototypical cases for the exhibition of ECBs in polyelectronic atoms. We start by stressing that the ECBs yield information not only about the frequency of oscillation but also about the wave-function characteristics of the timedependent superposition for each system of interest. These ought to leave their fingerprints on the results of the probe step of the overall pump-probe delay measurement. This feature is analogous to situations in time-independent spectroscopies (e.g., high resolution photoelectron spectroscopy), where the combination of experimental and theoretical information leads to the recognition and interpretation of statespecific and excitation-specific properties and processes.

The previous statement is clearly supported by the following simple consideration. Let us assume that a twocomponent time-dependent superposition is projected onto an arbitrary vector, $|u\rangle$. In general, the overlaps are nonzero. Specifically, suppose we consider the Al case [see Eq. (5) below], and write the superposition of an autoionizing state of complex energy $z_{1}=E_{1}-i \frac{1}{2} \Gamma$, and a discrete state of real energy $E_{2}$, which is created at the end of the pump stage, as $\Psi(t)=c_{1} e^{-i z_{1} t}\left|\psi_{1}\right\rangle+c_{2} e^{-i E_{2} t}\left|\psi_{2}\right\rangle(\hbar=1) . \Gamma$ is the width of the resonance state. Let the occupation amplitudes be $c_{1}$ $=\rho_{1} e^{-i \varphi_{1}}$ and $c_{2}=\rho_{2} e^{-i \varphi_{2}}$, and the overlaps of each component with $|u\rangle$ be $s_{1}=\left\langle u \mid \psi_{1}\right\rangle$ and $s_{2}=\left\langle u \mid \psi_{2}\right\rangle$. Then, the corresponding survival probability is

$$
\begin{aligned}
P(t) \equiv & |\langle u \mid \Psi(t)\rangle|^{2}=e^{-\Gamma t} \rho_{1}^{2} s_{1}^{2}+\rho_{2}^{2} s_{2}^{2} \\
& +2 e^{-(\Gamma / 2) t} \rho_{1} s_{1} \rho_{2} s_{2} \cos \left[\left(E_{2}-E_{1}\right) t+\left(\varphi_{2}-\varphi_{1}\right)\right] .
\end{aligned}
$$

The expression (3) refers to a possible result from the timeresolved probing of the superposition. Therefore, the last term contains information not only about the frequency but also about the wave-function constitution. In principle, the qualitative and quantitative features of this information could be explored by comparing the theoretical results to possible pump-probe measurements.

(a) The four-electron ionic state $\mathrm{N}^{3+} 1 s^{2} 2 s 2 p{ }^{1} P^{0}$. The initial state is the $\mathrm{N}^{3+} 1 s^{2} 2 s 2 p{ }^{1} P^{0}$ excited state, which is assumed prepared, say via the electric dipole transition $\mathrm{N}^{3+} 1 s^{2} 2 s^{2}{ }^{1} S \rightarrow 1 s^{h} 2 s 2 p{ }^{1} P^{0}$. Two pulses interact with it so as to produce transitions downwards, to $1 s^{2} 2 s^{2}{ }^{1} S$, and upwards to the position of $1 s^{2} 2 p^{2}{ }^{1} S$ as follows:

$$
\left.\mathrm{N}^{3+} 1 s^{2} 2 s 2 p^{1} P^{0}\right|_{+h \nu^{\prime}(\text { pulse }) \rightarrow \mathrm{N}^{3+}} ^{+h \nu\left(s^{2} 2 p^{2}{ }^{1} S^{\prime}\right.} .
$$

It is the consequence of the nonstationary superposition of the $1 s^{2} 2 s^{2}{ }^{1} S$ and $1 s^{2} 2 p^{2}{ }^{1} S$ configurations that has been studied here. Contrary to the previous case of $\mathrm{He}[1-3]$, where the two excited states are autoionizing, here the ECB involves two discrete states, of which one is multiply excited and the other is the ground state. As before, it is found that, as prepared, these nonstationary states also undergo ECBs in the attosecond range. The choice of an ion, i.e., of a system with an increased effective nuclear charge, was made so as to have a spectrum that matches better the large photon energies, which are associated with the production of attosecond pulses from high energy harmonic spectra. In the scheme (4), the experimental [19] photon energies of the simultaneously applied pulses are 16.20 and $12.98 \mathrm{eV}$, respectively.

We end by recognizing that any future experiment aiming at combining hyperfast pump-probe delay techniques with beams of atomic ions will have to use pulses whose intensity is sufficient to overcome the difficulties created by the low density of such beams. Perhaps, pulses from new sources, such as free-electron lasers, may prove suitable.

(b) The thirteen-electron bound state $\mathrm{Al} K L 3 s 3 p^{2}{ }^{2} P$. For this system, we have studied aspects of the excitation and correlation of the three-electron valence electrons of $\mathrm{Al}$ in the self-consistent field of the core electrons. The initial state Al $K L 3 s 3 p^{2}{ }^{2} P$ is reachable from the ground state Al $K L 3 s^{2} 3 p^{2} P^{0}$, say via photoabsorption. It lies $8400 \mathrm{~cm}^{-1}$ above the $\mathrm{Al}^{+} K L 3 s^{2}$ threshold [19]. Yet, symmetry restrictions forbid its autoionization and so it belongs to the discrete spectrum. The excitation-deexcitation scheme induced by the pulses is

$$
\left.\mathrm{Al} K L 3 s 3 p^{2} 2\right|_{+h \nu^{\prime}(\text { pulse }) \rightarrow \mathrm{Al} K L 3 s^{2} 3 p^{2} P^{0}} ^{+h \nu\left(\text { pulse } p^{3} P^{0}\right.} .
$$

Of the two states that are excited coherently, one is discrete (the ground state) and one is autoionizing. For the latter, which is labeled by the doubly excited $K L 3 p^{3}{ }^{2} P^{0}$ configuration, no previous knowledge as to its existence and properties existed. It turns out that it has a very short autoionization lifetime, of the order of $10^{-15} \mathrm{~s}$, which is appropriate for considerations concerning hyperfast (a few hundreds of attoseconds) measurements.

(ii) Coherent time-dependent excitation and decay of inner-hole states. Continua with one and two free electrons. Application to Al. Let us assume that we are dealing with a neutral atom. Pulses of high photon energies can photoeject an electron from an inner subshell of an atomic state. The inner-hole excited configuration is normally in the atom's continuous spectrum of one or more free electrons, and therefore it gives rise to one or more autoionizing states, $L S$ coupled or otherwise. Even if it is a single inner electron that is ejected, the overall physics is affected by the different structures, self-consistent fields, and electron correlation effects of initial and final states. For properties described in the time-independent framework, this fact was demonstrated decades ago, although their accurate computation is still a demanding problem.

Things become more interesting, and more complicated, when these processes are viewed through the prism of timedependent coherent excitation and evolution. A particular feature of the corresponding physics, i.e., the Auger decay, was recently time resolved in the pioneering XUV pumplaser probe experiments of Drescher et al. [20] on Krypton.

From the point of view of theory, the phenomenon of the time-resolved Auger decay was first computed from first principles for a few prototypical nonstationary atomic states about a decade ago [21]. In that work, as with all theoretical work on decaying states, it was assumed that decay is decoupled from the excitation step. On the other hand, the coherent 
time-resolved excitation and exponential decay is present in the predictions of $[1,2]$ as well as in the present work.

For a fast pump-probe delay interaction time and a short autoionization lifetime, the consequent physics must have some dependence on the coherent superposition of the relevant amplitudes of excitation (ionization) and decay. In the simplest case, the open channels involve one- as well as two-electron continua. On the other hand, in the timedependent coherent excitation-decay process in helium that was first studied in [1,2], there is no two-electron continuum.

Recently, Kazansky and Kabachnik [22] presented results of calculations on aspects of the coherent process of ionization decay of the Ne $1 s$ shell. (The ionization energy is $870 \mathrm{eV}$ [23], i.e., it is too large for the current attosecond pulse technology). The theoretical treatment was based on approximating in various ways the relevant accurate wave functions and the solution of the coupled time-dependent equations. Apart from the lack of good orbital functions and of electron correlation in initial and final states, one of the approximations used in [22] is the neglect of the direct ionization into the two-electron continuum into which the Auger state is embedded and of the exploration of the effects of the consequent amplitude superpositions. The theory and computations that were implemented via the SSEA in the present work, have solved the problems that are related to the above complexities with sufficient accuracy. Actually, in the next section we report results showing the effect of neglecting the direct excitation to the two-electron continuum.

As a system which is computationally tractable and which might be a good candidate for experimental work since the energy differences are realistic, and which involves an open - rather than a closed-(sub)shell initial state, we chose the following (combination of A,B,C):

(A) $\mathrm{Al} 1 s^{2} 2 s^{2} 2 p^{6} 3 s^{2} 3 p^{2} P^{0}+\left.h \nu \rightarrow\right|_{\mathrm{Al}^{+} 1 s^{+} 2 s 22 p^{6} 3 s^{2} 3 p^{1} P^{0}+\varepsilon_{2} p} ^{\mathrm{A}^{+} p^{2} 26^{6} s^{2} p^{3}{ }^{0}+\varepsilon_{1} p}$,

(B) $\mathrm{Al} 1 s^{2} 2 s^{2} 2 p^{6} 3 s^{2} 3 p^{2} P^{0}+h \nu \rightarrow \mathrm{Al}^{2+}\left[1 s^{2} 2 s^{2} 2 p^{5} 3 s^{2}{ }^{2} P^{0} \varepsilon \ell \ell^{1,3} P^{0}\right] \varepsilon^{\prime} p^{2} L, \quad \ell=s, d, \quad{ }^{2} L={ }^{2} S,{ }^{2} P,{ }^{2} D$

(C) $\mathrm{Al}^{+} 1 s^{2} 2 s 2 p^{6} 3 s^{2} 3 p^{3,1} P^{0} \stackrel{\text { Auger decay }}{\longrightarrow} \mathrm{Al}^{2+} 1 s^{2} 2 s^{2} 2 p^{5} 3 s^{2}{ }^{2} P^{0}+\hat{\varepsilon} \ell$.

The scheme $(A, B, C)$ represents a possible coherent sequence of inner-hole creation via ionization of the $2 s$ electron by an electromagnetic pulse and symmetry-restricted decay into only two Auger channels, the $\mathrm{Al}^{2+} 1 s^{2} 2 s^{2} 2 p^{5}\left({ }^{2} P^{0}\right)$ $\times\left(3 s^{2}{ }^{1} S\right) \varepsilon s, \varepsilon d^{1,3} P^{0}$, caused by the electron rearrangement $(2 p, 3 p) \rightarrow(2 s, \hat{\varepsilon} \ell), \ell=s, d$. Because of symmetry and electronic structure, this sequence is much less complex than other possible ones, such as the ones involving a $2 p$-hole state or electron pair rearrangements of the type $2 p 3 s \leftrightarrow 2 s \varepsilon p$ (rather than the $2 p 3 p \leftrightarrow 2 s \varepsilon d$ one). The assumption that is made in carrying out the relevant calculations is that the magnitudes of the essential physics is not altered by interchannel coupling, which is therefore left out.

In process (A), the $2 s$ subshell of the ground state is ionized by the electromagnetic pulse. In the electric dipole approximation, two final $L S$-coupled core states are reached, each with its own self-consistent field and its own correlation effects. (The approximation which is often made in various types of calculations, namely, taking the same orbital and $\mathrm{N}$-electron functions for such multiplet terms, contains errors). The corresponding scattering orbitals are labeled by $\varepsilon_{1} p$ and $\varepsilon_{2} p$ and are computed as term-dependent functions in the fixed HF potential of the core.

In process (B), the same pulse ejects two electrons, one occupying the $3 p$ orbital and one occupying the $2 p$ orbital. The pairs of uncorrelated orbitals representing the two free electrons are labeled by $\left(\varepsilon d, \varepsilon^{\prime} p\right)$. Other combinations of orbital angular momenta for scattering orbitals are left out, for reasons of computational economy. The resulting twoelectron continuum is degenerate with the one created by (A) and the subsequent Auger process $(\mathrm{C})$. In process $(\mathrm{C})$, the Auger electron orbital is labeled by $\hat{\varepsilon} \ell=\hat{\varepsilon} s, \hat{\varepsilon} d$.

For the problem of interest here, the evolution is driven by the coherent time-dependent superposition of the amplitudes for (A), (B), and (C). As already stated, additional excitation-decay channels could, in principle, also be included in $\Psi_{\text {SSEA }}(t)$. However, the calculation would become huge, requiring considerably larger computational power, without altering significantly either the physics that is discussed here or the essence of the theory and its methods.

\section{RESULTS OF CALCULATIONS}

If, in attempting to solve the TDSE, the normal type of CI with common basis sets were envisioned for each state (wave functions and energies), the overall calculations for the schemes of Sec. III would seem formidable (if not impossible), especially for the inner electron ionization in $\mathrm{Al}$, even if the formalism for the incorporation of the scattering function spaces were available. However, things are made much simpler and transparent in the context of the SSEA, and the apparent size of calculation is reduced to acceptable levels by considering three factors.

The first has to do with the fact that even at the zero order level the wave functions are optimized in a state-specific way. As a result, orbital relaxation and multiplet structure are 
accounted for. The small price one pays for this is the fact of nonorthonormality (NON) of orbitals of the same symmetry belonging to different configurations. On the other hand, explicit consideration of the NON that is due to the different self-consistent fields facilitates convergence of the overall calculation as well as the efficient computation of electron correlation.

The second is the fact that the purpose of this calculation is not to obtain quantities such as total energies with detailed accuracy, since the physical relevance of such an objective as regards the dynamics of the specific channels would be weak.

The third factor follows from the previous two. It has to do with the fact that by drawing from the experience of calculations of electronic structures and stationary state properties, e.g., [6-10], it is easy to understand a priori and anticipate the identity of the few configurations that play the major role for the problems of interest. For example, for the old problem of the ab initio calculation of electric dipole absorption oscillator strengths, where the requirements for accuracy of the matrix element as compared to the experimental accuracy are rather high, it is known since the early 1970s that it is not necessary to obtain very accurate solutions of the TISE for initial and final states for the calculation of the transition moment to be accurate. Instead, what is needed is to obtain the correct coefficients of the most important for the transition process configurations, which are constructed from state-specific orbitals $([8,9]$, and references therein).

It is in this spirit that the breakdown and reduction of the TDMEPs treated in this work were applied. The first important step is to decide on the types of amplitudes that are needed, so as to have the correct terms in the trial expansions in Eq. (1). Then follows the calculation of only the major characteristics of each wave function that is used in the expansion. This means that the calculation of the state-specific wave functions (2), which enter in the SSEA, may involve either one or only a few symmetry-adapted configurations, rather than a large expansion that is needed for a very accurate calculation of the total energy.

\section{A. Results for (a)}

The state-specific wave functions for the case of $\mathrm{N}^{3+} 1 s^{2} 2 s 2 p^{1} P^{0}$ were calculated via the MCHF method. Only six configurations were included, since the interest here is in the main configurations representing each state and in the energy differences. The important element in the calculation is the numerical accuracy of the orbitals and of the CI coefficients. These are not affected significantly by additional correlation configurations that are important for the total energy.

Since the objective for the SSEA calculation of the ECBs is to engage the main configurations only, the results of the state-specific computations show that the relevant wave functions are well represented by the following two-term expressions:

$$
\begin{gathered}
\Psi_{\text {initial }}\left({ }^{1} P^{0}\right)=0.992\left(1 s^{2} 2 s 2 p\right)-0.122\left(1 s^{2} 2 p 3 d\right)+\cdots, \\
\Psi_{\text {ground }}\left({ }^{1} S\right)=0.964\left(1 s^{2} 2 s^{2}\right)+0.263\left(1 s^{2} 2 p^{2}\right)+\cdots, \\
\Psi_{\text {excited }}\left({ }^{1} S\right)=0.959\left(1 s^{2} 2 p^{2}\right)-0.264\left(1 s^{2} 2 s^{2}\right)+\cdots .
\end{gathered}
$$

The calculated energy differences (from the six-configuration MCHF results), which were used in the overall SSEA calculations, are $\hbar \omega=16.52 \mathrm{eV}$ and $\hbar \omega^{\prime}=13.06 \mathrm{eV}$. (As already stated, the experimental ones are $\hbar \omega=16.20 \mathrm{eV}$ and $\hbar \omega^{\prime}$ $=12.98 \mathrm{eV}$ ).

The polarization of the two laser fields was chosen to be linear, along the $z$ axis. The field strength is, in a.u.,

$$
F(t)=g(t)\left[F \cos (\omega t)+F^{\prime} \cos \left(\omega^{\prime} t\right)\right],
$$

where $g(t)$ is the envelope of the pulse, having the trapezoidal shape

$$
g(t)= \begin{cases}\frac{t}{T_{\text {rise }}}, & 0 \leq t \leq T_{\text {rise }}, \\ 1, & T_{\text {rise }} \leq t \leq T_{\text {rise }}+T_{\text {flat }}, \\ 1-\frac{t-T_{\text {rise }}-T_{\text {flat }},}{T_{\text {off }}}, & T_{\text {rise }}+T_{\text {flat }} \leq t \leq T_{\text {rise }}+T_{\text {flat }}+T_{\text {off }} .\end{cases}
$$

According to the SSEA, the time-dependent solution of the TDSE is written as

$$
\left.\Psi_{\text {SSEA }}(t)=x_{\text {in }}(t)\left|\Psi_{\text {initial }}^{{ }^{1} P^{0}}\right\rangle+x_{\text {gr }}(t) \mid \Psi_{\text {ground }}^{1} S\right\}+x_{\text {exc }}(t)\left|\Psi_{\text {excited }}^{1} S\right\rangle .
$$

The above time-dependent coefficients are obtained by solving the coupled equations subject to the initial conditions $x_{i n}(t=0)=1, x_{g r}(t=0)=x_{\text {exc }}(t=0)=0$.

Figure 1 depicts the time-dependent occupation probabilities for the configurations $1 s^{2} 2 s^{2}$ and $1 s^{2} 2 p^{2}{ }^{1} S$, during and after the laser-ion interaction period. The pulse duration was chosen to be $152 \mathrm{fs}$, with $T_{\text {rise }}=T_{\text {off }}=12 \mathrm{fs}$, and the intensity was $I=8.75 \times 10^{9} \mathrm{~W} / \mathrm{cm}^{2}$. 


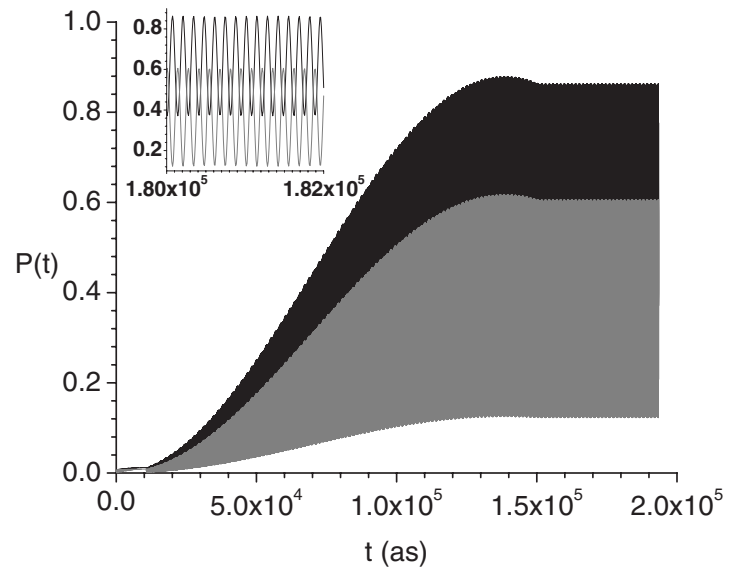

FIG. 1. Time-dependent occupation probabilities $P(t)$ of the $\mathrm{N}^{3+} 1 s^{2} 2 s^{2}{ }^{1} S$ and $1 s^{2} 2 p^{2}{ }^{1} S$ configurations during and after the laser-ion interaction. The configurations are excited from the $\mathrm{N}^{3+} 1 s^{2} 2 s 2 p{ }^{1} P^{0}$ state by two pulses of duration $152 \mathrm{fs}$, with intensity $I=8.75 \times 10^{9} \mathrm{~W} / \mathrm{cm}^{2}$ and frequencies $\omega=16.52 \mathrm{eV}, \omega^{\prime}$ $=13.06 \mathrm{eV}$. The black and gray curves correspond to the $1 s^{2} 2 s^{2}{ }^{1} \mathrm{~S}$ and $1 s^{2} 2 p^{2}{ }^{1} S$ configurations, respectively. The inset depicts clearly, for a short period after the laser-ion interaction, the oscillations of the occupation probabilities between the configurations.

In accordance with our earlier results on the strongly correlating ${ }^{1} P^{0}$ doubly excited states of $\mathrm{He}[1,2]$, the oscillation period between the strongly correlated configurations $1 s^{2} 2 s^{2}$ and $1 s^{2} 2 p^{2}{ }^{1} S$ is of the order of only 134 as. This fact can be read off the inset of Fig. 1, where the occupation probabilities after the laser ion interaction period are shown. The wave function and frequency information from these oscillations could in principle be recorded by projecting the interacting configurations on scattering states or, on the bound $2 s 3 p, 2 p 3 s, 2 p 3 d^{1} P^{0}$ states, where, due to orbital orthogonalities, only the first is accessible via the $2 s^{2}$ configuration and the other two via the $2 p^{2}$ one. To this end, the probe pulses should be in the attosecond scale and in harmony with the variation of the pump-probe time delay.

\section{B. Results for (b)}

The main configurations of the three states involved in the Al $K L 3 s 3 p^{2}{ }^{2} P$ scheme were

$$
\Psi_{\text {initial }}\left({ }^{2} P\right)=0.963\left(K L 3 s 3 p^{2}\right)-0.199\left(K L 3 p^{2}{ }^{1} D\right) 3 d+\cdots,
$$

$$
\Psi_{\text {ground }}\left({ }^{2} P^{0}\right)=0.979\left(K L 3 s^{2} 3 p\right)-0.201\left(K L 3 p^{3}\right)+\cdots
$$

$$
\Psi_{\text {autoion }}\left({ }^{2} P^{0}\right)=0.950\left(K L 3 p^{3}\right)+0.165\left(K L 3 s^{2} 3 p\right)+\cdots,
$$

where the corresponding energy differences from the initial bound state are 0.2473 a.u. and 0.2282 a.u.

A comment on the autoionizing state $\mathrm{Al} 1 s^{2} 2 s^{2} 2 p^{6} 3 p^{3}{ }^{2} P^{0}$ is in order here. To the best of our knowledge, this doubly excited state has not been observed or computed before. Our calculations show that it is a resonance state, lying about $2.3 \mathrm{eV}$ above the $K L 3 s 3 p{ }^{3} P^{0}$ threshold of $\mathrm{Al}^{+}$. In order to carry out the solution of the TDSE for the present purposes, we obtained its position as well as its width. The theory and methodology that were implemented for the computation of these properties followed our earlier work, e.g., [13]. The first step is the state-specific calculation of the localized wave packet $\Psi_{0}$ and of its energy $E_{0}$. The energy is computed variationally subject to suitable constraints and to the satisfaction of the virial theorem, and is determined from its optimal position in a local minimum inside the continuous spectrum. Given the objective of the overall calculations, only the essential electron correlation in the three valence electrons was computed. The main MCHF configurations were the $3 p^{3}, 3 s^{2} 3 p$, and $3 p 3 d^{2}$. To these, singly and doubly excited configurations were added, with optimized virtual orbitals up to $f$ symmetry.

The information on the decay of this resonance state to the $\mathrm{Al}\left(K L 3 s 3 p^{3} P^{0}\right) \varepsilon d^{2} P^{0}$ and $\left(K L 3 s 3 p^{3} P^{0}\right) \varepsilon s^{2} P^{0}$ continua could have been obtained within the SSEA to the solution of the TDSE, by including explicitly the scattering wave functions, as it was demonstrated in our previous papers $[1,2]$. However, for reasons of computational economy we chose to use in the state-specific expansion a complex energy, by adding to $E_{0}$ an imaginary part representing the decay width. The width was calculated from the energydependent golden rule-type formula used previously (see Eq. (14) of $[13(\mathrm{a})]$ ), assuming the independent channel approximation. Going beyond a first order (energy-independent) golden rule-type calculation, requires taking into account the recoupling of the higher roots of ${ }^{2} P^{0}$ symmetry, which is caused by the interaction with the open channel. These roots result from the diagonalization of the Hamiltonian in the space of ${ }^{2} P^{0}$ wave functions. As a consequence, the mixing coefficients in the expansion of the $\Psi_{\text {autoion }}\left({ }^{2} P^{0}\right)$ wave function become energy dependent. As anticipated from the electron correlation rule of symmetric exchange of orbital symmetry (SEOS), e.g., [8,9,24,14], the contribution of the $\varepsilon s$ channel is about two orders of magnitude smaller than that of the $\varepsilon d$ channel. The result for the width is $\Gamma=0.0145$ a.u., implying that this state has the very short lifetime of about 1.7 fs.

Regarding now the SSEA calculation, the polarization of the two laser fields was chosen to be linear, along the $z$ axis. Their temporal shape is as in Eqs. (7) and (8), with $\omega$ $=0.2473$ a.u. and $\omega^{\prime}=0.2282$ a.u.

The calculated oscillations of the occupation probabilities of the $K L 3 p^{3}{ }^{2} P^{0}$ and $K L 3 s^{2} 3 p^{2} P^{0}$ configurations, during and after the laser-ion interaction period, are shown in Fig. 2. They have a period of 320 as, i.e., the effect of electron correlation in this case is weaker than in the $\mathrm{Ne}^{3+}$ case. The oscillations diminish quickly as time elapses, due to the very short lifetime of the $K L 3 p^{3}{ }^{2} P^{0}$ autoionizing state.

For the overall process to be efficient, the electromagnetic pulses must be short compared to the lifetime of the autoionizing state. Indeed, the pulse was chosen to have a duration of 605 as with $T_{\text {rise }}=T_{\text {off }}=121$ as. In addition, the pulse has to be sufficiently strong. In our case, $I=8.75 \times 10^{11} \mathrm{~W} / \mathrm{cm}^{2}$, which is larger than the currently produced intensities of attosecond pulses $[25,26]$. We recognize that, for higher inten- 


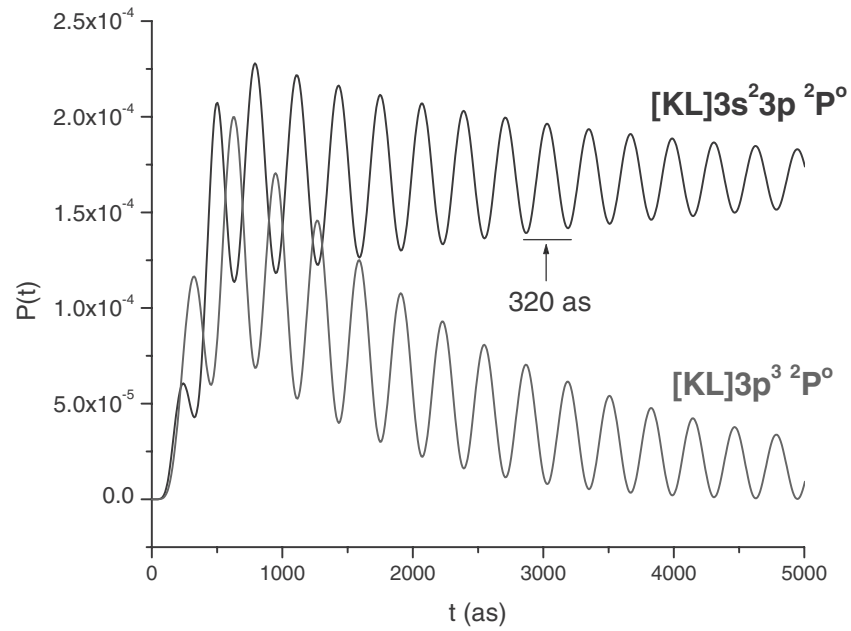

FIG. 2. Time-dependent occupation probabilities $P(t)$ of the Al $K L 3 s^{2} 3 p$ and $K L 3 p^{3}{ }^{2} P^{0}$ configurations during and after the laser-ion interaction. The configurations are excited from the $K L 3 s 3 p^{2}{ }^{2} P$ discrete state of $\mathrm{Al}$ by two pulses of duration 605 as with intensity $8.75 \times 10^{11} \mathrm{~W} / \mathrm{cm}^{2}$ and frequencies $\omega=6.73 \mathrm{eV}, \omega^{\prime}$ $=6.21 \mathrm{eV}$. The black and gray curves correspond to the $K L 3 s^{2} 3 p$ and $K L 3 p^{3}{ }^{2} P^{0}$ configurations, respectively. The latter configuration labels an autoionizing state, for which calculations show its position at about $2.3 \mathrm{eV}$ above the $K L 3 s 3 p^{3} P^{0}$ threshold of $\mathrm{Al}^{+}$with a lifetime of $1.7 \mathrm{fs}$.

sities, where the population transfer from the initial state will be much larger than the $2 \%$ that corresponds to the above intensity, the physically significant processes may involve a larger number of $\mathrm{Al}$ states, including scattering ones. Nevertheless, the present calculations do reveal quantitatively the essence of aspects of the time-dependent physics of excitation and of electron correlations and autoionization decay in strongly correlated states of polyelectronic states.

Again, as in the previous case, the information from these oscillations could in principle be observed by projecting via hyperfast pump-probe delay the nonstationary state on a higher lying resonance state. Given that the attosecond pulses have large frequencies, such a state would likely correspond to a configuration with a hole in the $2 s$ or the $2 p$ subshells. The overall measurement would then involve the observation of emission of electrons from the inner-hole state.

For process (ii) of Sec. III, in order to render the overall calculation attractive from the point of view of computational effort, we had to reduce its overall magnitude while keeping the parts that produce the important information for this problem. To this effect, each physically significant wave function entering in the expansion (1) was represented by a state-specific HF function, thereby omitting terms representing the localized electron correlation of each state. Of course, due to the Hamiltonian interaction matrix elements, a variety of CI effects are automatically accounted for, while solving the TDSE via the SSEA, including those causing the autoionization $(2 p, 3 p) \rightarrow(2 s, \hat{\varepsilon} d)$.

Therefore, based on the combination $(\mathrm{A}, \mathrm{B}, \mathrm{C})$ of Sec. III, the form of the $\Psi_{S S E A}(t)$ was

$$
\begin{aligned}
\Psi_{S S E A}(t)= & a(t) \Phi_{0}\left({ }^{2} P^{0}\right)+\int_{0} d \varepsilon \sum_{L} C_{3}^{L}(\varepsilon, t)\left[\Phi_{0}^{i o n}\left({ }^{3} P^{0}\right) \varepsilon p^{2} L\right] \\
& +\int_{0} d \varepsilon \sum_{L} C_{1}^{L}(\varepsilon, t)\left[\Phi_{0}^{i o n}\left({ }^{1} P^{0}\right) \varepsilon p^{2} L\right] \\
& +\iint_{0} d \varepsilon d \varepsilon^{\prime} \sum_{L} D_{3}^{L}\left(\varepsilon, \varepsilon^{\prime}, t\right) \\
& \times\left[\left(\Phi_{0}^{i o n}\left({ }^{2} P^{0}\right) \varepsilon d\right)^{3} P^{0} \varepsilon^{\prime} p^{2} L\right] \\
& +\iint_{0} d \varepsilon d \varepsilon^{\prime} \sum_{L} D_{1}^{L}\left(\varepsilon, \varepsilon^{\prime}, t\right) \\
& \times\left[\left(\Phi_{0}^{i o n}\left({ }^{2} P^{0}\right) \varepsilon d\right)^{1} P^{0} \varepsilon^{\prime} p^{2} L\right]
\end{aligned}
$$

where $\Phi_{0}\left({ }^{2} P^{0}\right), \Phi_{0}^{i o n}\left({ }^{3} P^{0}\right), \Phi_{0}^{i o n}\left({ }^{1} P^{0}\right)$, and $\Phi_{0}^{i o n}\left({ }^{2} P^{0}\right)$ are numerical $\mathrm{HF}$ functions for the $\mathrm{Al} K L 3 s^{2} 3 p^{2} P^{0}$, $\mathrm{Al}^{+} 1 s^{2} 2 s 2 p^{6} 3 s^{2} 3 p^{3} P^{0}, \quad \mathrm{Al}^{+} 1 s^{2} 2 s 2 p^{6} 3 s^{2} 3 p^{1} P^{0}, \quad$ and $\mathrm{Al}^{2+} 1 s^{2} 2 s^{2} 2 p^{5} 3 s^{2}{ }^{2} P^{0}$ configurations, respectively. The corresponding energy differences from the ground state $\Phi_{0}\left({ }^{2} P^{0}\right)$ are, 4.686 a.u., 4.701 a.u., and 3.457 a.u.

The label $L$ stands for $S, P$, and $D$ symmetries. The energy-normalized scattering orbitals are obtained numerically in the frozen core HF approximation. The orbitals $\varepsilon^{\prime} p$ and $\varepsilon d$ were obtained from two independent computations, the first in the field of $\Phi_{0}^{i o n}\left({ }^{1,3} P^{0}\right)$ and the second in the field of $\Phi_{0}^{i o n}\left({ }^{2} P^{0}\right)$. No account for electron correlation in the twoelectron continuum produced by direct ionization was taken. This type of possible interaction cannot change the essence of the herein calculated phenomena.

The Hamiltonian has the form

$$
H(t)=H_{\text {atom }}+V(\omega, t),
$$

where the field-atom interaction for the linearly polarized pulse is taken as

$$
V(\omega, t)=z F g(t) \sin (\omega t)
$$

$g(t)$ has the trapezoidal temporal shape. The values of the field strength $F$ were taken to range from the weak-field limit, where perturbation theory holds, to higher values where large population transfer takes place from the $\mathrm{Al}$ ground state.

The nonperturbative solution of the TDSE

$$
\frac{\partial}{\partial t} \Psi_{S S E A}(t)=\left[H_{\text {atom }}+V(\omega, t)\right] \Psi_{S S E A}(t)
$$

is obtained by first converting the linear TDSE into a system of coupled integrodifferential equations [use of expansion (11)]. The numerical method of solution is an appropriate generalization of the Taylor expansion technique [6] that allowed for the incorporation of the double continuum.

The $\mathrm{Al}^{+} 2 s$-hole state decays into the continua $1 s^{2} 2 s^{2} 2 p^{5} 3 s^{2} \varepsilon \ell^{1,3} P^{0}, \ell=s$ or $d$, during and after the interaction with the laser pulse and the matrix elements $\left\langle\Phi_{0}^{\text {ion }}\left({ }^{1,3} P^{0}\right) \varepsilon p^{2} L\left|H_{\text {atom }}\right|\left(\Phi_{0}^{\text {ion }}\left({ }^{2} P^{0}\right) \varepsilon \ell\right)^{1,3} P^{0} \varepsilon^{\prime} p^{2} L\right\rangle$ account for autoionization. In the spirit of the SSEA, the terms representing the $2 p 3 p \leftrightarrow 2 s \varepsilon s$ rearrangement were not included in the final calculations since, for the ${ }^{1} P^{0}$ channel, which is 

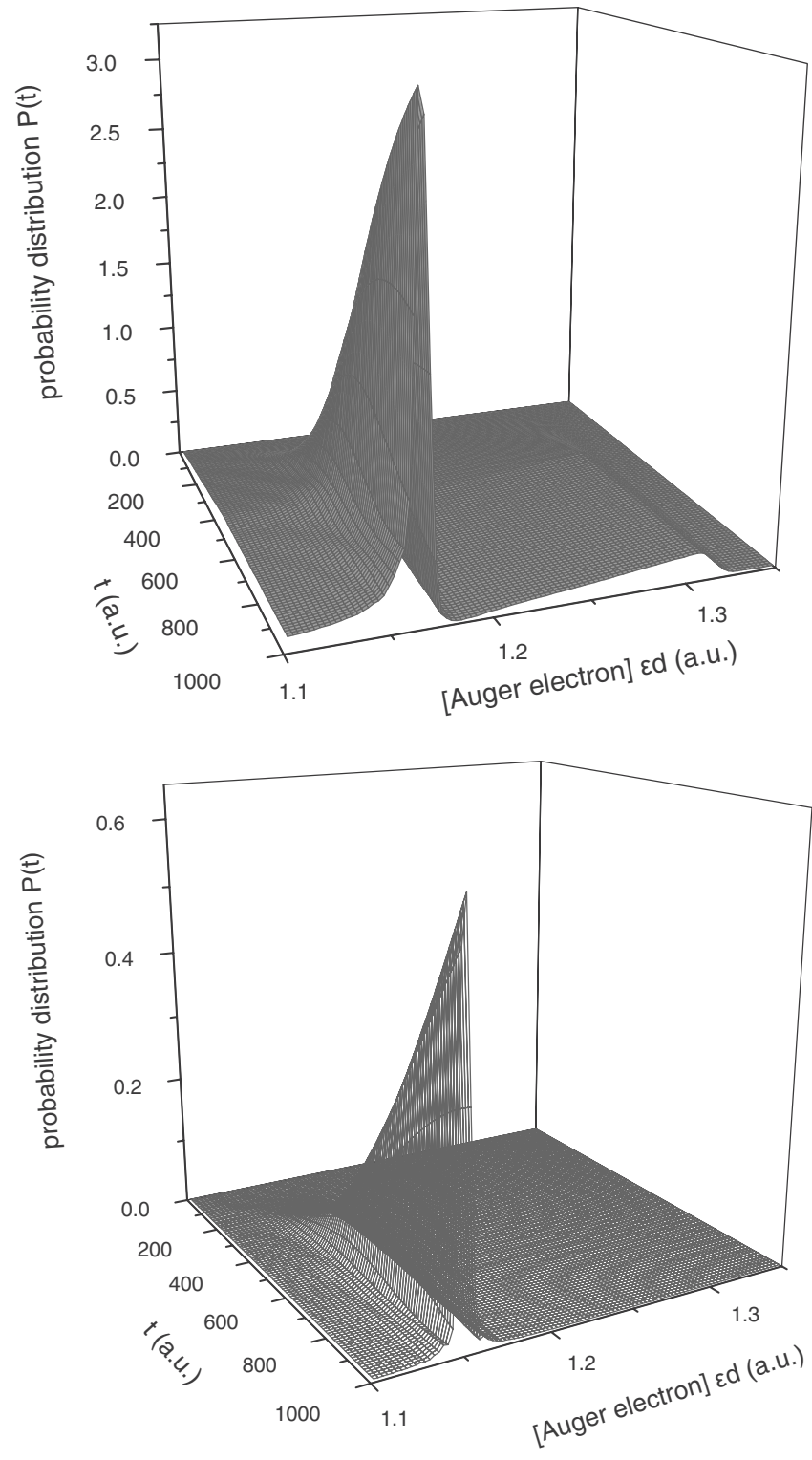

FIG. 3. (a) Integrated, over the energy $\varepsilon^{\prime}$ of the $\varepsilon^{\prime} p$ scattering orbital, probability distribution of the $\mathrm{Al}\left(1 s^{2} 2 s^{2} 2 p^{5} 3 s^{2} \varepsilon d\right)^{1} P^{0} \varepsilon^{\prime} p$ continuum states as a function of energy $\varepsilon$ and of time $t$. The $\mathrm{Al}$ ground state ${ }^{2} P^{0}$ interacts with a trapezoidal laser pulse of duration 450 a.u. (10.9 fs), frequency $\omega=4.851$ a.u. $(132 \mathrm{eV})$, and intensity $8.75 \times 10^{11} \mathrm{~W} / \mathrm{cm}^{2}$. For this intensity, the percentage of the initial state population that ionizes is very small, only $0.02 \%$. The Auger energy distribution exhibits a Fano-type asymmetry with peak values at $31.7 \mathrm{eV}$. (b) As in (a), but for the $\mathrm{Al}\left(1 s^{2} 2 s^{2} 2 p^{5} 3 s^{2} \varepsilon d\right)^{3} P^{0} \varepsilon^{\prime} p$ continuum states. The Auger energy distribution is essentially symmetric.

the most important [see Figs. 3(a) and 3(b)], the corresponding matrix elements are one order of magnitude smaller than the $2 p 3 p \leftrightarrow 2 s \varepsilon d$ ones.

In spite of the justifiable reductions to which we have already referred, the system of coupled equations that must be solved contains about one million state-specific terms, the main requirement coming from the double electron continuum. For example, the number of terms in the system of coupled equations that results from the expansion (11) is of the order of one million, a fact which renders the numerical problem nearly intractable. This obstacle was overcome via the approximation that is discussed in the Appendix. Specifically, by taking advantage of the energy degenerate states corresponding to the different total angular momenta ${ }^{2} S,{ }^{2} P$, and ${ }^{2} D$, we transform linearly the time-dependent coefficients, with the result that it is necessary to integrate a system of "only" about 300000 coupled equations.

Figure 3(a) shows the integrated (over the energy $\varepsilon^{\prime}$ of the $\varepsilon^{\prime} p$ scattering orbitals), differential ionization probability of the $\mathrm{Al}\left(1 s^{2} 2 s^{2} 2 p^{5} 3 s^{2} \varepsilon d\right)^{1} P^{0} \varepsilon^{\prime} p$ continuum states as a function of the energy $\varepsilon$ and of time $t$, arising from the interaction of the $\mathrm{Al}$ ground state $K L 3 s^{2} 3 p^{2} P^{0}$ with a trapezoidal laser pulse of duration 450 a.u. (10.9 fs) with $T_{\text {rise }}=T_{\text {off }}=94$ as. The frequency $\omega$ of the pulse is 4.8508 a.u. $(132 \mathrm{eV}), 4.1 \mathrm{eV}$ above the $2 s$-holestate $\left(1 s^{2} 2 s 2 p^{6} 3 s^{2} 3 p\right)^{1} P^{0}$. This figure represents the first ab initio theoretical-computational demonstration of the time-resolved formation of the interference-induced asymmetric profile during the creation of an inner-hole Auger state [3,5].

At this point, it is appropriate to comment on the possible presence of postcollision interaction (PCI), e.g., [27]. This is a special type of electron pair correlation effect in the continuous spectrum, whose significance has been known to be important when the energy of the ejected photoelectron is very close to threshold, say in the range $0-2 \mathrm{eV}$. Although the exact energy range for PCI to influence the observables depends on the system and on the property, it is reasonable to assume that for photoelectron energies of $\sim 4.1 \mathrm{eV}$ and an Auger electron energy of $31.7 \mathrm{eV}$, as is the case in our problem, PCI cannot play an important role. We point out that the inclusion of the PCI in the solution, from first principles, of the TDSE for polyelectronic atoms in laser fields has not been achieved yet. On the other hand, future experiments could possibly measure not only the time-resolved formation of the asymmetric Fano profile, but also the effect that the PCI has on this, by choosing appropriate ionization frequencies.

The results for the $\mathrm{Al}\left(1 s^{2} 2 s^{2} 2 p^{5} 3 s^{2} \varepsilon d\right)^{3} P^{0} \varepsilon^{\prime} p$ continuum states are shown in Fig. 3(b). In this case, the time-resolved interference-induced profile is essentially symmetric. A main reason for the difference between the two channels as regards the profile is the following: In the case of the ${ }^{3} P^{0}$ channel, the interference of the independent paths to the twoelectron continuum, which in this case is the generating mechanism of the asymmetric Fano profile, is not significant. This is corroborated by the numerical fact that the electric dipole matrix elements to the two-electron continuum in the ${ }^{3} P^{0}$ channel are two orders of magnitude smaller than the ones for the ${ }^{1} P^{0}$ channel.

Figure 4 shows the differential ionization probability of the $\mathrm{Al}$ continuum states, as a function of energy $\varepsilon$ and of time $t$, for the process $\mathrm{Al} K L 3 s^{2} 3 p^{2} P^{0}+h \nu$ (pulse) $\rightarrow \mathrm{Al}^{+}\left(1 s^{2} 2 s 2 p^{6} 3 s^{2} 3 p\right)^{1} P^{0} \varepsilon p$. The exponential decay is caused, mainly, by the strong interaction of the $\mathrm{Al}^{+} 2 s$-hole state $\left(1 s^{2} 2 s 2 p^{6} 3 s^{2} 3 p\right)^{1} P^{0}$, with the continuum states $\mathrm{Al}^{2+}\left(1 s^{2} 2 s^{2} 2 p^{5} 3 s^{2}\right)^{2} P^{0} \varepsilon d$. The autoionization lifetime that corresponds to this exponential decay is $5.7 \mathrm{fs}$.

The results given in Figs. 3(a) and 4 were obtained for the magnetic quantum number $M=0$. In order to complete the 


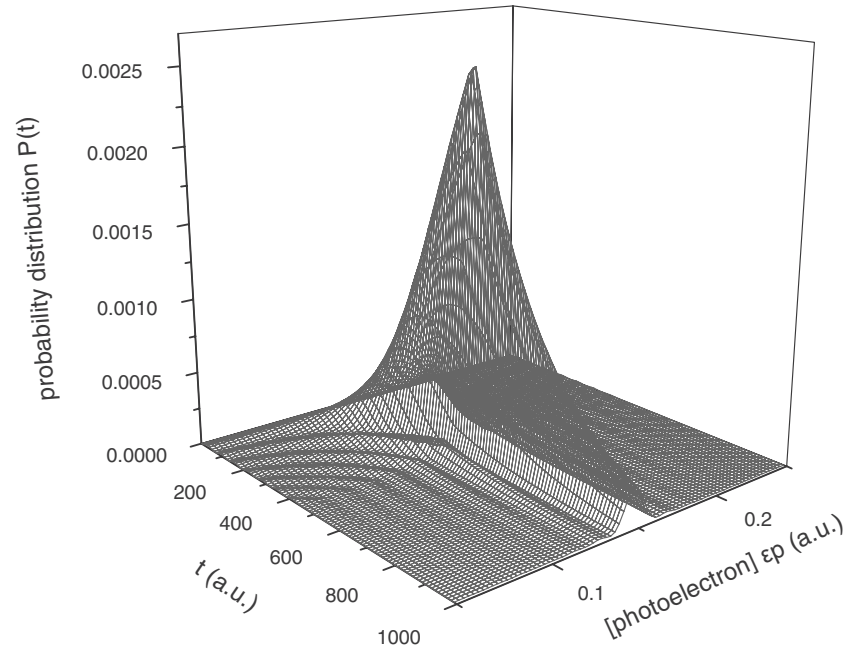

FIG. 4. Probability distribution of the $\mathrm{Al}$ continuum states $\mathrm{Al}^{+}\left(1 s^{2} 2 s 2 p^{6} 3 s^{2} 3 p\right)^{1} P^{0}+$ photoelectron orbital $\varepsilon p$, as a function of energy $\varepsilon$ and of time $t$. The laser pulse characteristics are as those of Fig. 3(a). The exponential decay of this distribution yields a lifetime of $5.7 \mathrm{fs}$ for the $1 s^{2} 2 s 2 p^{6} 3 s^{2} 3 p^{1} P^{0} \mathrm{Al}^{+} 2 s$-hole state.

picture, we have also obtained results for $M= \pm 1$. These are shown in Figs. 5 and 6. It is seen that they are qualitatively similar to those of Figs. 3(a) and 4, apart from small quantitative differences.

Concerning the issue of the two-electron continuum, we also performed calculations similar in spirit to those of Kazansky and Kabachnik [22], meaning that the channel of the direct ionization to the two-electron continuum into which the Auger state is embedded was neglected. Figure 7 shows the differential ionization probability of the Al $\left[\left(1 s^{2} 2 s^{2} 2 p^{5} 3 s^{2}\right)^{2} P^{0} \varepsilon d\right]^{1} P^{0} \varepsilon^{\prime} p$ continuum states as a function of the energy $\varepsilon$ and of time $t$, after integration over the energy $\varepsilon^{\prime}$ of the $\varepsilon^{\prime} p$ scattering orbital has been carried out. It

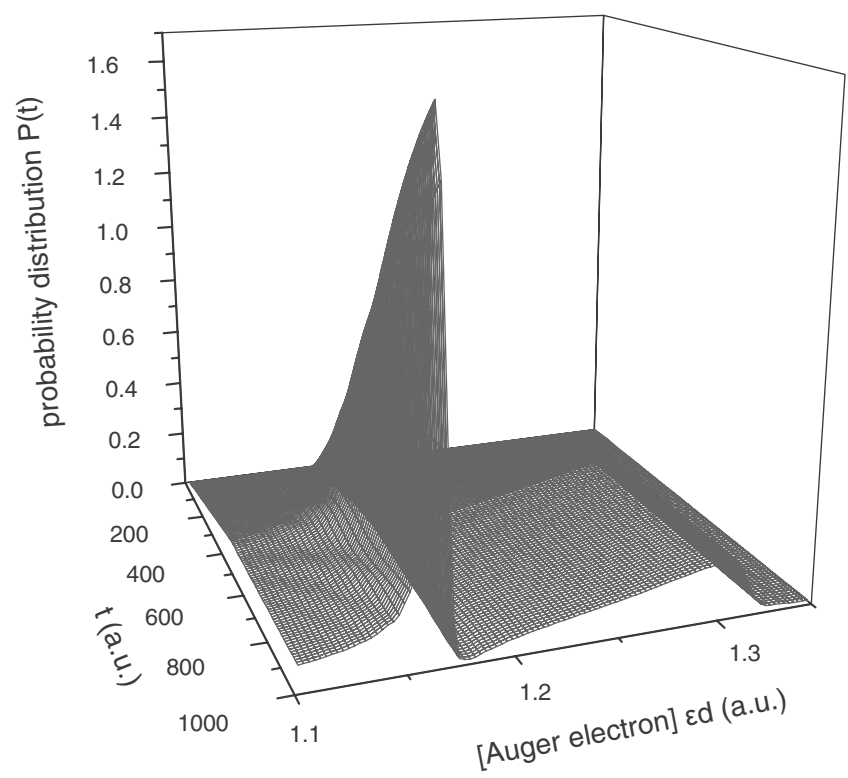

FIG. 5. As in Fig. 3(a), but for magnetic quantum number $M$ $= \pm 1$.

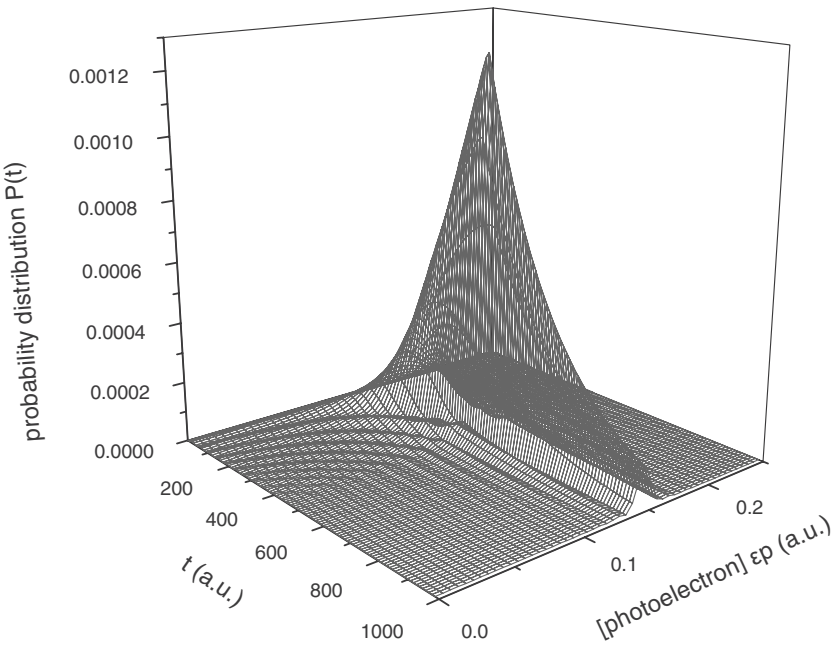

FIG. 6. As in Fig. 4, but for magnetic quantum number $M$ $= \pm 1$.

is clear that, in addition to quantitative differences, there are qualitative differences that alter the physical picture. Specifically, the time-dependent formation of the asymmetric profile, which is clear in Fig. 5, is not observed in Fig. 7. This is because the asymmetric profile is the consequence of the amplitude superposition of the independent ionization paths to the $\mathrm{Al}\left[\left(1 s^{2} 2 s^{2} 2 p^{5} 3 s^{2}\right)^{2} P^{0} \varepsilon d\right]^{1} P^{0} \varepsilon^{\prime} p$ continuum states.

\section{CONCLUSION}

Given the currently developing frontier of time-resolved spectroscopy with hypershort electromagnetic pulses, the present work focused on the solution of prototypical time-

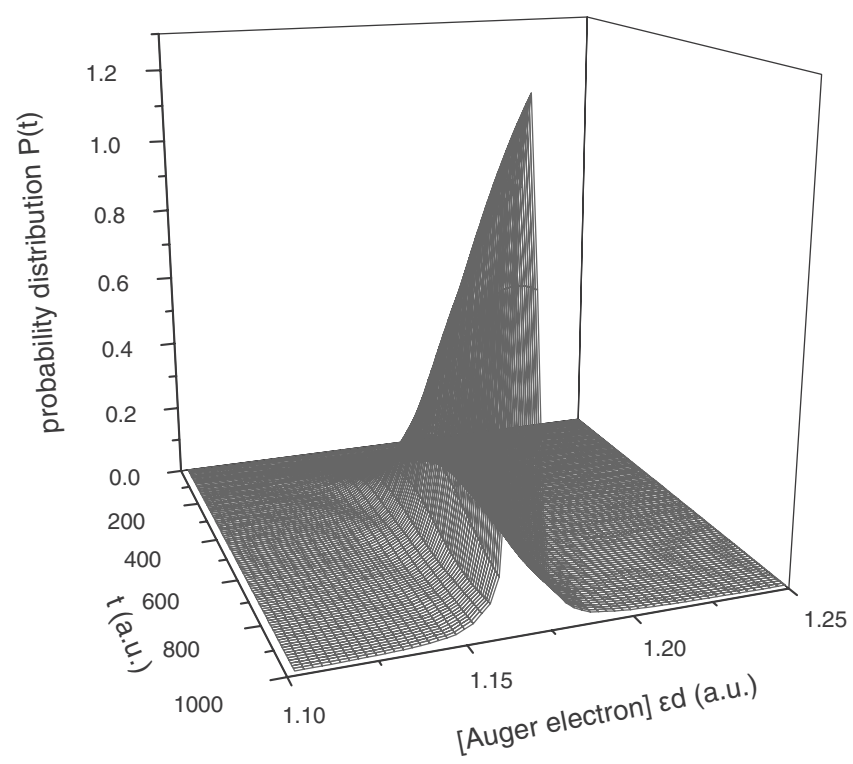

FIG. 7. Integrated, over the energy $\varepsilon^{\prime}$ of the $\varepsilon^{\prime} p$ continuum orbital, probability distribution of the $\mathrm{Al}\left(1 s^{2} 2 s^{2} 2 p^{5} 3 s^{2} \varepsilon d\right)^{1} P^{0} \varepsilon^{\prime} p$ continuum states as a function of energy $\varepsilon$ and of time $t$. In this calculation, the direct ionization to the two-electron continuum is neglected. 
dependent problems involving the excitation and decay of excited states in real, polyelectronic atoms, such as $\mathrm{Al}$ and $\mathrm{N}^{3+}$. The emphasis was on producing quantitative results for time-resolved effects that result from strong electron correlation. For example, phenomena such as electron correlation beats (ECBs), where information on wave functions is revealed [Eq. (3)], and excitation and decay of inner subshell Auger states, have been calculated from first principles. The solution of the corresponding time-dependent Schrödinger equations was achieved by implementing the state-specific expansion approach, introduced in [6] and further reviewed here. In the present case, the size of the expansion reached, after appropriate reductions (see Appendix ), more than 300000 terms.

For the study of ECBs, which occur within time scales of a few hundreds of attoseconds, two characteristic systems (atom plus two pulses) were examined [see processes (4) and (5)].

For the problem of the coherent excitation and decay of the $2 s$-hole Auger states labeled by $\mathrm{Al}^{+}\left(1 s^{2} 2 s 2 p^{6} 3 s^{2} 3 p\right)^{1,3} P^{0}$, the contribution of both the oneand the two-electron scattering continua were included in the calculation of quantities that are sensitive to amplitude interference, such as the time-dependent formation of the autoionization profile [3,5], which, in the case of the ${ }^{3} P^{0}$ channel is essentially symmetric and in the case of the ${ }^{1} P^{0}$ channel exhibits a Fano-type asymmetry.

\section{ACKNOWLEDGMENT}

This work was supported by the program "Pythagoras," which is co-funded by the European social fund $(75 \%)$ and national resources $(25 \%)$.

\section{APPENDIX}

Here we explain and justify the reduction mentioned in the text of the (huge) number of coupled differential equations that result when the $\left(1 s^{2} 2 s 2 p^{6} 3 s^{2} 3 p\right)^{1,3} P^{0} \varepsilon^{\prime} p$ and $\left(1 s^{2} 2 s^{2} 2 p^{5} 3 s^{2} \varepsilon d\right)^{1,3} P^{0} \varepsilon^{\prime} p$ continuum states with total angular momenta ${ }^{2} S,{ }^{2} P,{ }^{2} D$ are included in the expansion.

The approximation is based on the assumption of decoupling of the autoionization channel for each symmetry, which is essentially determined by the matrix element $\langle 2 p 3 p|H| 2 s \varepsilon d\rangle$ from the rest. Once this channel is initially excluded, then the system of equations for the excitation of the $\mathrm{Al}$ ground state $1 s^{2} 2 s^{2} 2 p^{6} 3 s^{2} 3 p^{2} P^{0}$ is written as

$$
\begin{aligned}
i \frac{d a(t)}{d t}= & E_{0} a(t)+F(t) \sum_{L=0}^{2} \int d \varepsilon V^{(L)}(\varepsilon) C^{(L)}(\varepsilon, t) \\
& +F(t) \sum_{L=0}^{2} \iint d \varepsilon d \varepsilon^{\prime} V^{(L)}\left(\varepsilon, \varepsilon^{\prime}\right) D^{(L)}\left(\varepsilon, \varepsilon^{\prime} ; t\right),
\end{aligned}
$$

$$
\begin{aligned}
i \frac{d C^{(L)}(\varepsilon, t)}{d t}=\left(E_{1}+\varepsilon\right) & C^{(L)}(\varepsilon, t)+F(t) V^{(L)}(\varepsilon) a(t), \quad L=0,1,2, \\
i \frac{d D^{(L)}\left(\varepsilon, \varepsilon^{\prime} ; t\right)}{d t}= & \left(E_{2}+\varepsilon+\varepsilon^{\prime}\right) D^{(L)}\left(\varepsilon, \varepsilon^{\prime} ; t\right) \\
& +F(t) V^{(L)}\left(\varepsilon, \varepsilon^{\prime}\right) a(t), \quad L=0,1,2,
\end{aligned}
$$

where $E_{0}, E_{1}$, and $E_{2}$ are the energies of the $\mathrm{Al} 1 s^{2} 2 s^{2} 2 p^{6} 3 s^{2} 3 p^{2} P^{0}, \quad \mathrm{Al}^{+}\left(1 s^{2} 2 s 2 p^{6} 3 s^{2} 3 p\right)^{1,3} P^{0} \quad$ and $\mathrm{Al}^{2+} 1 s^{2} 2 s^{2} 2 p^{5} 3 s^{2}{ }^{2} P^{0}$ states correspondingly. $F(t)$ symbolizes the time-dependent linearly polarized electromagnetic pulse and $V^{(L)}(\varepsilon)$ and $V^{(L)}\left(\varepsilon, \varepsilon^{\prime}\right)$ are the dipole interaction matrix elements between the $\mathrm{Al}$ ground state and the continuum states with $L=0,1,2$.

We now define the new time-dependent coefficients

$$
\begin{gathered}
C(\varepsilon, t)=\sqrt{\sum_{L=0}^{2}\left[V^{(L)}(\varepsilon)\right]^{2} \sum_{L=0}^{2} V^{(L)}(\varepsilon) C^{(L)}(\varepsilon, t),} \\
D\left(\varepsilon, \varepsilon^{\prime} ; t\right)=\sqrt{\sum_{L=0}^{2}\left[V^{(L)}\left(\varepsilon, \varepsilon^{\prime}\right)\right]^{2} \sum_{L=0}^{2} V^{(L)}\left(\varepsilon, \varepsilon^{\prime}\right) D^{(L)}\left(\varepsilon, \varepsilon^{\prime} ; t\right) .}
\end{gathered}
$$

After a few algebraic steps, we obtain the new, size-reduced, and to a large extent term-independent system of integrodifferential equations

$$
\begin{aligned}
& i \frac{d a(t)}{d t}= E_{0} a(t)+F(t) \int d \varepsilon \sqrt{\sum_{L=0}^{2}\left[V^{(L)}(\varepsilon)\right]^{2} C(\varepsilon, t)} \\
&+F(t) \sum_{L=0}^{2} \iint d \varepsilon d \varepsilon^{\prime} \sqrt{\sum_{L=0}^{2}\left[V^{(L)}\left(\varepsilon, \varepsilon^{\prime}\right)\right]^{2}} D\left(\varepsilon, \varepsilon^{\prime} ; t\right), \\
& i \frac{d C(\varepsilon, t)}{d t}=\left(E_{1}+\varepsilon\right) C(\varepsilon, t)+F(t) \sqrt{\sum_{L=0}^{2}\left[V^{(L)}(\varepsilon)\right]^{2}} a(t), \\
& i \frac{d D\left(\varepsilon, \varepsilon^{\prime} ; t\right)}{d t}=\left(E_{2}+\varepsilon+\varepsilon^{\prime}\right) D\left(\varepsilon, \varepsilon^{\prime} ; t\right) \\
&+F(t) \sqrt{\sum_{L=0}^{2}\left[V^{(L)}\left(\varepsilon, \varepsilon^{\prime}\right)\right]^{2} a(t)}
\end{aligned}
$$

The new time-dependent coefficients correspond to the new continuum states, consisting of linear combinations of states with different total angular momenta, as in Eq. (A2). The conjecture is that the inclusion in the final calculation of the autoionization channel does not affect significantly the numerical results stemming from the transformation (A2). 
[1] C. A. Nicolaides, Th. Mercouris, and Y. Komninos, J. Phys. B 35, L271 (2002)

[2] Th. Mercouris, Y. Komninos, and C. A. Nicolaides, Phys. Rev. A 69, 032502 (2004).

[3] Th. Mercouris, Y. Komninos, and C. A. Nicolaides, Phys. Rev. A 75, 013407 (2007).

[4] S. X. Hu and L. A. Collins, Phys. Rev. A 71, 062707 (2005).

[5] M. Wickenhauser, J. Burgdörfer, F. Krausz, and M. Drescher, Phys. Rev. Lett. 94, 023002 (2005); J. Mod. Opt. 53, 247 (2006).

[6] Th. Mercouris, Y. Komninos, S. Dionissopoulou, and C. A. Nicolaides, Phys. Rev. A 50, 4109 (1994).

[7] C. A. Nicolaides, Phys. Rev. A 6, 2078 (1972).

[8] Articles by D. R. Beck and C. A. Nicolaides, in Excited States in Quantum Chemistry, edited by C. A. Nicolaides and D. R. Beck (Reidel, Dordrecht, 1978).

[9] C. A. Nicolaides, Int. J. Quantum Chem. 60, 119 (1996); C. A. Nicolaides, ibid. 102, 250 (2005).

[10] Y. Komninos and C. A. Nicolaides, Z. Phys. D: At., Mol. Clusters 4, 301 (1987); Phys. Rev. A 34, 1995 (1986); J. Phys. B 37, 1817 (2004).

[11] Although here we use the nonrelativistic atomic Hamiltonian, the same concepts regarding the choice and optimization of state-specific function spaces apply either with the Breit-Pauli approximation with one- and two-electron spin-dependent operators, e.g., [24,14], or with the four component Dirac Hamiltonian, e.g., $[28,23,29]$. The wave functions of such a type of calculation have the SoC form, and so are suitable for the SSEA formalism that is used with the nonrelativistic wave functions.

[12] C. Froese-Fischer, Comput. Phys. Commun. 14, 145 (1978).

[13] (a) Y. Komninos, G. Aspromallis, and C. A. Nicolaides, Phys. Rev. A 27, 1865 (1983); (b) C. A. Nicolaides, N. A. Piangos, and Y. Komninos, ibid. 48, 3578 (1993); (c) C. A. Nicolaides and N. A. Piangos, Phys. Rev. A 64, 052505 (2001), and references therein.

[14] Y. Komninos, G. Aspromallis, and C. A. Nicolaides, J. Phys. B 28, L423 (1995).

[15] M. J. Seaton, Rep. Prog. Phys. 46, 167 (1983), and references therein.
[16] T. Brage, C. Froese-Fischer, and N. Vaeck, J. Phys. B 26, 621 (1993).

[17] G. N. Bates, Comput. Phys. Commun. 8, 220 (1974).

[18] Y. Komninos, Th. Mercouris, and C. A. Nicolaides, Phys. Rev. A 65, 043412 (2002); 71, 023410 (2005); Th. Mercouris, Y. Komninos, and C. A. Nicolaides, J. Phys. B 35, 1439 (2002).

[19] Atomic Energy Levels, Natl. Stand. Ref. Data Ser., Natl. Bur. Stand. (U.S.) 35, Vol. I, edited by C. E. Moore (1949).

[20] M. Drescher, M. Hentschel, R. Kienberger, M. Ulberacker, V. Yakovlev, A. Scrinzi, Th. Westerwalbesloh, U. Kleineberg, U. Heinzmann, and F. Krausz, Nature (London) 419, 803 (2002).

[21] C. A. Nicolaides and Th. Mercouris, J. Phys. B 29, 1151 (1996); Th. Mercouris and C. A. Nicolaides, J. Phys. B 30, 811 (1997).

[22] A. K. Kazansky and N. M. Kabachnik, Phys. Rev. A 72 , 052714 (2005); J. Phys. B 39, L53 (2006).

[23] D. R. Beck and C. A. Nicolaides, in [8]. Article on "Theory of One Electron Binding Energies Including Correlation, Relativistic and Radiative Effects: Application to Free Atoms and Metals".

[24] G. Aspromallis and C. A. Nicolaides, J. Phys. B 19, L13 (1986); G. Aspromallis, Ch. Sinanis, and C. A. Nicolaides, J. Phys. B 29, L1 (1996).

[25] A. Baltuska, Th. Udem, M. Ulberacker, M. Hentschel, E. Goulemakis, Ch. Gohle, R. Holzwarth, V. S. Yakoviev, A. Scrinzi, T. W. Hansch, and F. Krausz, Nature (London) 421, 611 (2003).

[26] P. Agostini and L. F. DiMauro, Rep. Prog. Phys. 67, 813 (2004)

[27] V. Schmidt, Rep. Prog. Phys. 55, 1483 (1992); M. Yu Kuchiev and S. A. Sheinerman, J. Phys. B 18, L551 (1985); M. Borst and V. Schmidt, Phys. Rev. A 33, 4456 (1986); G. B. Armen, J. C. Levin, and I. A. Sellin, ibid. 53, 772 (1996); J. A. R. Samson, Y. Lu, and W. C. Stolte, ibid. 56, R2530 (1997).

[28] J. P. Desclaux, Comput. Phys. Commun. 9, 31 (1975).

[29] D. R. Beck and Z. Cai, Phys. Rev. A 41, 301 (1990); D. R. Beck, ibid. 57, 4240 (1998); L. Pan and D. R. Beck, J. Phys. B 39, 4581 (2006). 